\title{
Transforming Instructional Development: Online Workshops for Faculty
}

\section{Laurie Bellows}

University of Nebraska, Lincoln

\section{Joseph R. Danos}

Delgado Community College

Two vastly different institutions, the University of Nebraska, Lincoln and Delgado Community College, cooperated in the delivery of online faculty development workshops in syllabus construction. This chapter describes the experiences of a flagship university and an urban community college in employing electronic delivery of the same workshop content to their respective faculty members. It shares successful and unsuccessful strategies, nuts and bolts, and the discovery of an unexpected, pleasant irony: The technology that can separate and isolate us has the potential to bring us together, as though we were on electronic legs in a virtual Athenian agora.

\section{INTRODUCTION}

Technology is transforming higher education. For the most part, it has made our work easier; it has changed how we communicate and interact with our colleagues and our students, and in some cases, it is changing what we do in the classroom (Baldwin, 1998). Recent research indicates that instructors are increasingly using web-based resources in some form to supplement their teaching (Bonk, 2001). Likewise, there are increased online opportunities available to help faculty improve their instruction (Gillespic, 1998; Shea, 
Sherer, \& Kristensen, 2002). Shea, Sherer, and Kristensen (2002) note that the proliferation of technology use in the classroom-both on the ground and online-presents new challenges and opportunities for those of us in faculty development: expansion of services, continuous delivery of programs through asynchronous communication, easier access to information, and opportunities for collaboration across departments, divisions, and campuses. The most important opportunity, perhaps, is that technology creates "new avenues for faculty to learn together" (Shea, Sherer, \& Kristensen, 2002, p. 170).

This chapter describes how we took advantage of the many opportunities technology provides to plan, design, develop, and deliver an online workshop in syllabus construction for faculty. It explains how two highly diverse institutions, the University of Nebraska, Lincoln (UNL) and Delgado Community College in New Orleans, collaborated to deliver the workshop to their faculties. We begin with a brief overview of the learner-centered syllabus and provide a rationale for developing an online instructional development workshop for faculty in syllabus construction. Next, we describe the workshop: illustrating the process with activities, materials used in the workshop, and the assessment tool used to evaluate the workshop's effectiveness and usefulness. We will share our individual campus experiences in offering and delivering the workshop, as well as the lessons learned. Finally, we examine the benefits and drawbacks in facilitating online workshops for faculty members.

\section{The Learner-Centered Syllabus}

In their 1995 article describing a new paradigm for undergraduate education, Robert Barr and John Tagg distinguished between the traditional instruction paradigm and a new learning paradigm. In the instruction paradigm, teachers are subject matter experts who transmit information to students primarily through lectures. In the learning paradigm, students are more actively responsible for their learning, often learning through cooperative groups and collaborative activities. What makes the learning paradigm so powerful is the shift of responsibility from teacher to student. This shift allows teachers to be designers of the learning environment instead of transmitters of information. An important strategy for promoting student learning is the learner-centered syllabus.

The learner-centered syllabus is one of the many outcomes of the learning paradigm. A learner-centered syllabus shifts the focus away from what we teach to what students learn (Grunert, 1997). Just as the instruction paradigm emphasized subject-matter expertise, the traditional syllabus was designed for 
the content-centered classroom. It was, however, ineffective in helping students understand their roles and responsibilities as active learners.

The learner-centered syllabus focuses on student learning by providing more comprehensive information about the course and instructor expectations for student learning. At the very least, it includes clearly stated course objectives, a course outline identifying specific assignments and activities with corresponding due dates, and statements about class policies and procedures. In effect, it places students at the very center of the question: What do students need to know to get the most out of this learning experience?

According to Diamond (1998), a learner-centered syllabus should accomplish the following goals:

- Define students' responsibilities as learners

- Define your roles and responsibilities to students

- Provide a clear statement of course goals and student outcomes

- Establish standards and procedures for evaluation

- Familiarize students with course logistics

- Establish a pattern of communication between instructor and students

- Include difficult to obtain materials such as readings, complex charts, and graphs

Although these ideas are not revolutionary, many teachers may never have taken the time to redesign a course syllabus that includes such comprehensive information. Thus, one objective of the workshop was to provide participants an initial opportunity to develop learner-centered syllabi.

\section{Rationale}

Our rationale for developing an online workshop in syllabus construction was threefold. First, recent campus needs assessment and focus group discussions indicate that time is the primary barrier that keeps most faculty members from participating in traditional face-to-face instructional development activities like workshops, seminars, and discussion groups. However, cutting-edge technology now makes it possible for faculty to participate in such activities at their convenience. Constructed as an online class using the Blackboard courseware management system, this workshop creates an asynchronous environment for faculty participation, allowing enrolled individuals to select both 
the time and place for their attendance at the workshop, while still encouraging discussion, feedback, and interactivity.

Second, both the University of Nebraska, Lincoln and Delgado Community College use Blackboard as their campus-wide online delivery system. We wanted to provide faculty an opportunity to learn how to use Blackboard and thereby create an incentive for them to utilize Blackboard in their own teaching. To reach more faculty members through the elimination of the time and place barrier, we decided to use the medium to teach the medium while delivering the desired content.

Finally, although training for the use of Blackboard is available on both campuses, it emphasizes the technology, not the pedagogy. The workshop topic, syllabus construction, arose because of its universal application, nonthreatening content, and widespread need of improvement. Since many instructors may be unfamiliar with the components of a learner-centered syllabus, we viewed this workshop as an opportunity for faculty to develop or revise their own course syllabi. As a side product, we hoped participants would use the workshop to reflect on and engage in scholarly conversations about their teaching with their peers.

\section{An Asynchronous, Online Workshop: Constructing a Learner-Centered Syllabus}

\section{Workshop Structure}

Following basic principles of course design, we began by asking what we want workshop participants to be able to do at the end of the online workshop. Our primary goal was to help faculty develop a learner-centered syllabus that connected their course objectives, activities, assignments, and assessment. In this context, our objectives for the workshop were to help parricipants:

- Develop a course rationale that could be shared with students

- Write measurable course objectives

- Identify assignments and tests that support course objectives

- Construct a course outline

- Compose a learner-centered syllabus for the next semester

Online delivery of the workshop provided additional benefits, including the opportunity for participants to 1) meet and work with colleagues in an 
online environment, 2) learn how to use Blackboard, and 3) experience online learning from a students' perspective.

The workshop was based on Judith Grunert's book, The Course Syllabus (1997), and was conceptualized as an asynchronous, interactive learning experience spanning a three-week period. Using our objectives as a guide, we organized the workshop around eight modules (Figure 9.1). The workshop was designed to take participants through the process of syllabus development, from start to finish, with the goal of creating or revising one for the following semester. Each module begins with a lesson to focus participants' thinking, and is followed by supplementary reading materials (i.e., brief essays) and related activities or learning tasks.

\section{FIGURE 9.1}

\section{Learning Modules}

Module 1: Overview: The Learning Centered Syllabus

Module 2: Developing a Course Rationale

Module 3: Setting the Stage: What Are Your Instructional Objectives?

Module 4: Assignments and Assessments

Module 5: Constructing a Course Outline

Module 6: Instructional Approaches and Strategies for Active Learning

Module 7: Composing the Syllabus

Module 8: Summary and Evaluation

\section{Workshop Content}

Blackboard offers a number of features that helped us organize the workshop. In the syllabus component we provided an overview of the workshop, posted the workshop goals and objectives, and included some tips on how to succeed in the process. We also provided participants a series of "how to..." instruction sheets for how to use email, the digital drop box, engage in small group discussion, and download PDF files in Blackboard. The course documents section included the eight learning modules with corresponding lessons, handouts, and activities. 
The discussion board area allowed participants a place to meet socially in the Coffee Bean. In this area, we invited participants to "grab a cup of coffee, sit back, and talk to us!" Participants were encouraged to use this space to socialize, tell jokes, update each other on their family vacations, and just have fun. We also used the discussion board as a place for participants to share their course rationale statements with other workshop participants, post their lists of activities and assignments, and reflect on the teaching and learning process.

FIGURE 9.2

\section{The Discussion Board}

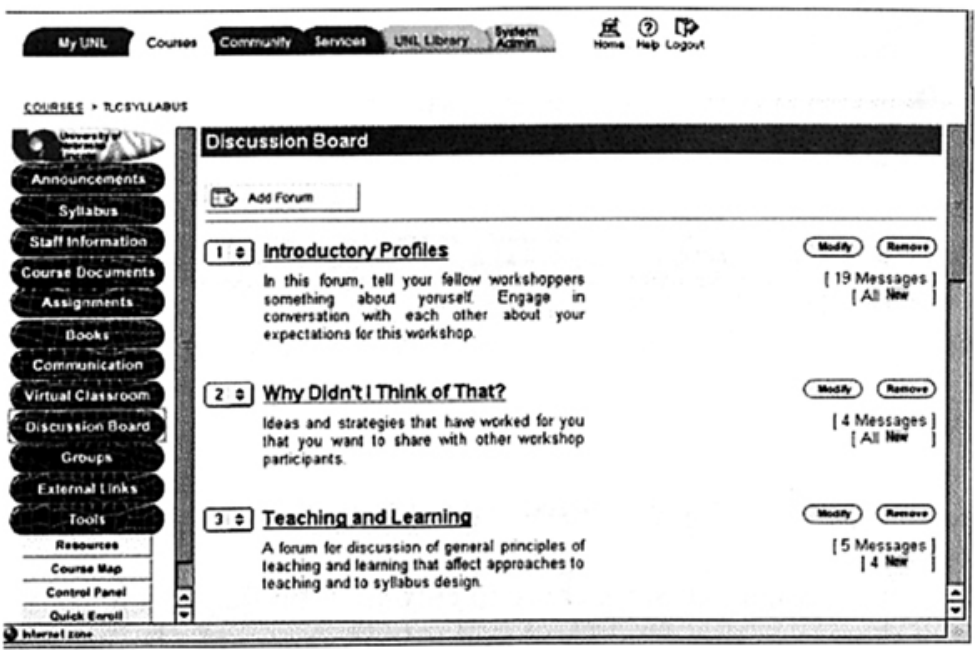

The excernal links feature provided participants additional resources on teaching and learning, including links to teaching and learning center sites in the United States and Canada.

Each module included three subfolders: focus your thinking, handouts, and your tasks (Figure 9.3). Module lessons contained brief overviews of the topic and questions to prompt reflections. For example, in Module 3, the importance of writing measurable and observable instructional objectives was explained, and participants were encouraged to think about 1) the kinds of skills and levels of knowledge they expected of students, 2) the level of performance they might expect from students, 3) the ways students would be "different" when they finished the course, and 4) what students should be able to do with the knowledge and skills gained in the course. 
FIGURE 9.3

Example of "Focus Your Thinking"

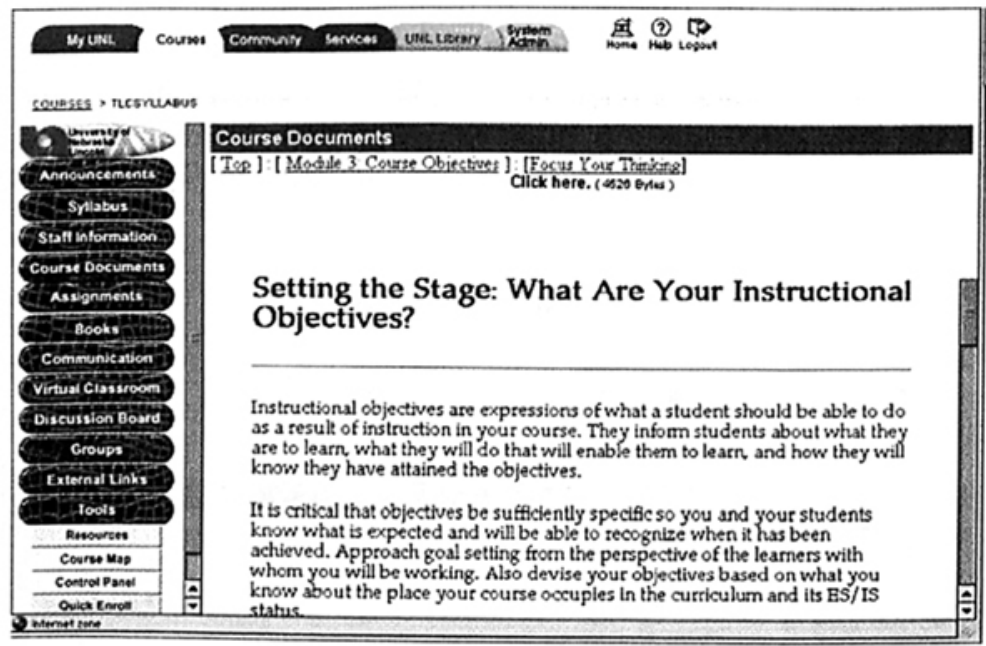

For each module, we identified various materials that would help supplement the lesson. We included essays, excerpts from our faculty instructional guide, and other text-based handouts used in previous face-to-face workshops.

In lieu of assignments, we chose to encourage participants to complete a series of short tasks that would lead to the development of a learner-centered syllabus. Each task was designed to help faculty critically examine the core assumptions about why they do what they do. In Module 2, for instance, we challenged participants to develop a course rationale that clearly communicates to their students their assumptions and expectations regarding their course. Faculty participants were asked to write a short memo describing the purpose of their course, explaining how it fits into their students' degree program and discussing how students will benefit from the course. The purpose of this task was to get participants engaged in scholarly reflection about their teaching. Once the rationale was drafted, participants were instructed to share the memo with their email buddies, and based on their feedback, rewrite the memo as a course rationale to include in their syllabus. We also created a forum for participants to share their course rationale with the entire workshop community. 
In the final module, putting it all together, faculty were expected to pull together each component of their syllabus and post the final product to the discussion forum for feedback and suggestions.

\section{Workshop Process}

A three-week online workshop was piloted at the University of Nebraska, Lincoln during June 2000. In fall 2000 and then again in summer 2001, the workshop was repeated within a five-week timeframe. The workshop also was offered at Delgado Community College in New Orleans in spring 2001. In the four versions of the workshop, "Constructing a Learner-Centered Syllabus," a total of 103 faculty members ( 78 from UNL and 25 from Delgado) participated.

University of Nebraska, Lincoln experience. During summer 2000, all faculty members and graduate teaching assistants were invited to participate via a flier in the three-week online workshop. Twenty-five registrations were received by email. Once participants were registered, they received a confirmation of their enrollment and a login ID, a password, and other tips for participating in an online workshop. In addition, each participant was assigned an email buddy to establish a sense of online community and to help answer questions or solve problems with the technology.

The three-week workshop began in mid-June. Participants were asked to post an introductory profile identifying themselves, their departments, the course for which they would be creating a syllabus, why they were drawn to the workshop, and their learning goals for the workshop. The remaining six modules were introduced about every third day, giving participants time to complete an assigned task, share their product with their email partners, revise their work, and then post it to the discussion forum for feedback. As new modules were posted, participants received an email reminding them that the module was ready for viewing. Announcements also were posted on the course page.

The workshop was offered again during the fall 2000 semester but based on post-workshop feedback from participants, we expanded it to a five-week format. Twenty-eight faculty members registered for the fall workshop. A third workshop was offered during a five-week session in summer 2001. Prior to the summer 2001 workshop, however, we offered a face-to-face orientation and included a hands-on introduction to Blackboard. Again, 25 faculty members registered for the third workshop.

Although the actual workshop was offered within a specific timeframe, only about one-third of all participants completed the workshop within the 
three- or five-week schedule, while approximately one-third of the participants completed the workshop on their own as a self-paced learning experience. The remaining one-third dropped out of the workshop and did not complete it.

Delgado Community College experience. In a spirit of collegiality, the University of Nebraska, Lincoln's Teaching and Learning Center agreed to share the content of its online workshop with Delgado Community College. The workshop was announced at the convocation that began Delgado's spring 2001 semester. Fifty-two of the 350 full-time faculty members filled out registration sheets indicating their desire to participate. However, the workshop was delayed until March 1 while we awaited the arrival of a new server to house it. By that time, 27 faculty members were no longer able to participate and the workshop began with 25 participants. Nine completed the entire workshop, posted syllabi to the discussion board, and received a $\$ 200$ stipend.

In recognition of the high demand placed on faculty time (the customary community college load of five courses and ten office hours per week produce a required 25-hour weekly presence on campus), we spaced the workshop over a ten-week period (March through mid-May). It began with a two-hour, hands-on introduction to Blackboard and new modules were posted every ten days. To encourage greater participation, four small groups were established, each with its own private discussion board area and virtual chat room (i.e., threaded discussion and synchronous chat). We also made use of the workshop's general discussion board area and the virtual classroom.

\section{Workshop Assessment and Outcomes}

Using the Blackboard assessment feature, we created an online survey to evaluate the workshop. Borh multiple choice and short-answer items were included. Additionally, several participants provided unsolicited feedback via email.

Although only about one-third of the total number of participants completed the workshop, we considered it a success on both campuses. Faculty feedback (see Table 9.1) indicated that both workshop goals were met. When asked if they had produced a syllabus as a result of their participation in this workshop, the majority of those completing the workshop evaluation indicated "yes" or "still working on it." 
TABLE 9.1

Workshop Assessment

$\mathbf{n = 3 2}$ (all four sessions)

SA $\quad$ A $\quad$ D $\quad$ SD

The workshop provided new and useful

information for my teaching.

$20 \quad 12$

The workshop adequately addressed my

\begin{tabular}{lrrrrr} 
questions about syllabus development. & 12 & 14 & 1 & 1 \\
\hline I achieved the goals I set for myself in this workshop. & 8 & 16 & 4
\end{tabular}

I would participate in another online

instructional development workshop.

\begin{tabular}{lllll}
\hline I plan to use Blackboard for teaching future courses. & 12 & 12 & 4
\end{tabular}

SA=Strongly Agree; $A=$ Agree; $D=$ Disagree; $S D=$ Strongly Disagree

The majority of those responding to the workshop evaluation indicated that the workshop had helped them think about their teaching and student learning. One participant wrote, "The workshop helped me review my syllabus with a critical eye and also provided some valuable information with which to refine the work in progress." Writing a course rationale, "creating course objectives that go beyond factual knowledge and stating them clearly," and learning "how to use Blackboard from a student's perspective" were considered the most important skills gained from the workshop.

We had also hoped that faculty participants would form an online community of learners and use the workshop as an opportunity to talk about their teaching with others. We purposely designed opportunities for them to share their materials and give feedback and suggestions to their peers. And initially they did. However, as the workshop progressed, discussion seemed to wane and there was very little conversation about teaching or syllabus construction. Yet, from the very beginning, participants expressed their pleasure as they explored the potential of this novel technological tool. It may be that discussing new technology is nonthreatening because everyone is a neophyte.

Based on the feedback, we concluded that the workshop fulfilled its primary objectives of helping faculty develop a learner-centered syllabus and stimulating faculty members to become acquainted with Blackboard. 


\section{LESSONS LEARNED}

Through participant feedback and our own observations, we have learned some important lessons about what worked, what did not (and why), and what we would do to improve the process.

\section{What Worked}

The workshop content was effective at an individual level. In general, participants found the workshop materials useful but, as noted above, the majority of the online discussion focused on how to use Blackboard. Through communications with individuals and the production and posting of syllabi, it was apparent that the participants were doing their homework by completing the assigned reading and tasks. However, they did not take the next step of engaging in meaningful discussion about their teaching. At several junctures in the workshop, participants were encouraged to provide feedback to their peers on the course rationale or to share the content of their course objectives, but they were hesitant to suggest changes. This hesitation probably arises from several factors, some of which include the novelty or unknown quality of the online environment, an unwillingness to be vulnerable with their peers, and a lack of trust of unknown peers (different campuses, different disciplines, etc.). The novelty factor will disappear as the participants become more comfortable using Blackboard. This factor is controllable by means of new online workshops, i.e., training, and the inception of user groups. The refusal to display professional vulnerability in a public medium will require a change in our institutional culture. We hope that the quasi-anonymity of the online environment will catalyze this change.

The method of delivery suited the content. At UNL, we originally offered this workshop in syllabus construction using the traditional face-to-face format. We scheduled two, two-hour sessions, weeks apart. During the first session we covered the background information on a learner-centered syllabus. The second session was scheduled in a computer lab so that participants could actually construct a syllabus in real time. However, the majority of faculty members who had participated in the first session did not return for the second session, citing lack of time as the major obstacle. In contrast, the online environment allowed us to present a contiguous learning experience while offering participants an opportunity to learn anytime, anywhere. Most important, the online environment allowed participants to construct the syllabus as they worked through the content of the workshop.

The online environment promotes networking. As an unexpected outcome of the workshop, we discovered that several faculty members from different 
campuses or disciplines met each other for the first time and formed potentially lasting relationships. Several participants suggested that we should have a faceto-face "wrap-up" meeting to end the workshop (a suggestion that we will incorporate into our next iteration). Although this may occur in any traditional campus-wide workshop, the probability of its occurrence is increased in an online workshop because more faculty members can participate in an asynchronous electronic workshop and the medium provides the opportunity for more individual and group communication (even if it is inirially on a superficial, mundane, "gee-whiz" level).

Collaborating with another institution. The considerable amount of time required to develop an online workshop ensures that collaboration across institutions will increase both from the allocation of scarce resources and the wheel's-been-invented standpoints. Along with the serendipitous sharing model described herein, opportunities for inter-institution collaboration abound: between different campuses of the same institution, different institutions in the same state, four-year (flagship) universities and community colleges in the same state, and institutions with no common geographical connection. Given the customary distribution of faculty development resources (i.e., more at flagship institutions, less at other four-year institutions, and almost none at community colleges), the most fertile field for collaboration exists between flagship institutions and community colleges. Of course, coequal institutions will also benefit from pooling their resources to produce and deliver online faculty development materials. We might expect, as well, that university administrators and state legislators will look with approval on this efficient expenditure of tax dollars.

If the two institutions use compatible online course delivery software, the workshop content is easily downloaded from one institution to the other. Since both Nebraska and Delgado utilize Blackboard, the transfer was uneventful in our case. Although Nebraska could have hosted the Delgado workshop from Lincoln, we decided to house the content on a Delgado server and to have a local facilitator. This arrangement was beneficial because it:

- Set a local tone that increased participation (increased comfort level with the known)

- Allowed faster response time for any technical problems

- Decreased the facilitation burden for Nebraska

- Decreased the technical support demands on Nebraska 
Following this model, a receiving institution could then host subsequent online workshops for sister institutions that have even fewer resources. For example, Delgado could now host online workshops (with acknowledgement of the source of the content) for the other community and technical colleges in Louisiana that are incapable of offering their own. We believe that this model and subsequent variations offer excellent potential for achieving an intellectual multiplier effect (for those of you with an economic bent) within a system and producing a concomitant reputation multiplier effect for the source institution-in this case, Nebraska. Nebraska's metaphorical bumblebee has pollinated magnolias on the bayou.

\section{What Did Not Work (and Why)}

The workshop pace. Whether it was offered as a three-week, a five-week, or a ten-week event, many participants found it difficult to complete the workshop within the scheduled timeframe. Some found that if they got behind, it was difficult to catch up, while others admitted to a lack of motivation, even with a stipend as an incentive to complete the workshop.

An invisible facilitator. Experts in online course delivery suggest that facilitators should be more of the guide on the side, so in keeping with good online practice, the instructional developers took a back seat in the workshop. For the most part, we were not visible in the workshop process. Participant feedback indicated that, perhaps, we should have taken a more facilitative role in the workshop such as posing discussion questions, providing feedback on completed tasks, and challenging participants to reflect on and discuss their teaching.

Buddy systems and small groups. Although the online learning literature suggests that assigning email buddies or dividing participants into small groups are good strategies for motivating and connecting online learners, we found that, overall, neither strategy produced greater participation in the workshop. At UNL, the small groups just never materialized. At Delgado, we found that every group had one or two more active participants who experienced more responses to their postings to the general workshop-wide discussion board than to those that they sent to their group's discussion area. Lack of participation in the small groups and posting to the discussion board in lieu of the small group discussion area may have been a function of each individual's time constraints. The end result was that the general discussion board contained the lion's share of the threaded discussions. If everyone had participated fully, the division of the workshop's participants into small groups should have worked. 


\section{Improving the Process}

Increase the facilitator's role. Online facilitators need to model and encourage a sense of community, at least at the beginning of the workshop. Their presence keeps the workshop participants focused and can serve as a motivator.

Provide incentives to complete the workshop. We believe it is important to reward faculty for their teaching improvement efforts. Participants suggested that, in lieu of a monetary award, a certificate of completion might be useful for their promotion and tenure files. We also learned that it would be helpful to establish deadlines for completion of individual tasks. As one participant noted, facilitators may need to be an "online nag" to keep folks engaged in the process.

Pace the workshop activities so that participants are not easily overwhelmed. We considered whether the workshop should be offered as a selfstudy activity. Though it is possible, such a format would emphasize the value of convenience over that of group involvement, communication, and peer-topeer instruction and sharing. It would preclude any institution-wide community building. We truly have no solution to this problem of workshop pace other than to note that instructional developers must realistically assess the amount of content that can be effectively delivered online in a given timeframe. In addition, participants should be encouraged to recognize that while learning online may be more convenient, it can also be more time-consuming than participating in a face-to-face workshop.

\section{Benefits and Drawbacks of Online Instructional DeVElopment Workshops}

There are a both benefits and drawbacks for instructional developers and faculty in delivering online instructional development workshops. From a faculty development perspective, the benefits include 1) providing a support system to help ease faculty transition into the use of technology, 2) helping faculty view the use of technology and the learning process from the students' vantage point, and 3) providing a vehicle for faculty to reflect on and discuss their teaching in a nonthreatening environment while encouraging interaction with their colleagues.

The benefits for faculty participants include 1) the asynchronous nature of online learning (any time, any place) makes it more convenient to engage in instructional development activities, and 2) the Blackboard discussion board area encourages conversation and dialogue that otherwise might not take place across disciplines, campuses, and possibly even institutions. 
Of course, with every benefit there is a drawback. For instructional developers, the major drawbacks of online instructional development include the up-front work involved in developing the workshop (i.e., many workshop materials may need to be redesigned because there is not necessarily a straight translation from face-to-face to online materials), the increased amount of FTE time focused on one event (i.e., up to one hour a day for three, five, or ten weeks versus a one-time, two-hour workshop), and the ability to respond to problems inherent in the use of technology (i.e., access to instructional technologists who can solve computer problems). The drawbacks for faculty participants are similar to students who engage in any form of online learning: staying motivated, keeping pace with the workshop, and feeling connected to your fellow learners you cannot see.

\section{Conclusion}

As the sense of technological novelty wanes and the cultural metamorphosis occurs, online instructional development workshops will help promote deeper intellectual exchange and discussion of pedagogical topics of broad import. The cross-campus, interdisciplinary relationships developed in this environment will strengthen the institutions. A pleasant irony is that the technology that can separate and isolate us has the potential to bring us together, as though we were on electronic legs in a virtual Athenian agora. Would it display too great hubris to say that we have the opportunity to play Socrates in transforming instructional development?

\section{REFERENCES}

Baldwin, R. G. (1998). Technology's impact on faculty life and work. In K. Herr Gillespie (Ed.), The impact of technology on faculty development, life and work (pp. 7-21). New Directions for Teaching and Learning, No. 76. San Francisco, CA: Jossey-Bass.

Barr, R. B., \& Tagg, J. (1995). From learning to teaching: A new paradigm for undergraduate education. Change, 27(6), 13-25.

Bonk, C. J. (2001). Online teaching in an online world. Bloomington, IN: CourseSHare.com.

Diamond, R. M. (1998). Designing and assessing courses and curricula: A practical guide. San Francisco, CA: Jossey-Bass. 
Gillespie, F. (1998). Instructional design for the new technologies. In K. Herr Gillespie (Ed.), The impact of technology on faculty development, life and work (pp. 39-52). New Directions for Teaching and Learning, No. 76. San Francisco, CA: Jossey-Bass.

Grunert, J. (1997). The course syllabus: A learner-centered approach. Bolton, MA: Anker.

Shea, T. P., Sherer, P. D., \& Kristensen, E. W. (2002). Harnessing the potential of online faculty development: Challenges and opportunities. In D. Lieberman \& C. Wehlburg (Eds.), To improve the academy: Vol. 20. Resources for faculty, instructionah and organizational development (pp. 162-178). Bolton, MA: Anker.

\section{Contact:}

Laurie Bellows

Faculty and TA Consultant

University of Nebraska

201 Benton Hall

Lincoln, NE 68588

Voice (402) 472-9764

Fax (402) 472-4932

Email lbellows1@unl.edu

Joseph R. Danos

Delgado Community College

2600 General Meyer Ave.

New Orleans, LA 70114

Voice (504) 361-6157

Email jdanos@dcc.edu

Lauric Bellows is with the Office of Graduate Studies at the University of Nebraska, Lincoln where she coordinates all centralized instructional support for graduate teaching assistants and collaborates with departments in discipline-specific TA training and development. She facilitates online workshops for faculty and teaches an online graduate course in college teaching and an undergraduate course in Educational Psychology.

Joseph R. Danos is an Associate Professor of Accounting at Delgado Community College. In his former position of Associate Dean of Business Studies, he introduced Blackboard to Delgado and oversaw the development and offering of 17 online courses for his division in three years. He has facilitated two online faculty development workshops at the college. 